Article

\title{
Nitrate Removal by Mixed-Bed Ion Exchange
}

Taekyung YOON, Gangchoon LEE

Department of Environmental and Chemical Engineering, Dongeui University, Gaya-dong 24, Pusanjin-ku, Pusan 614-714, Korea

Byeongil NOH

Department of Chemical Engineering, Dongseo University, Pusan 617-716, Korea

Byunghyun MOON

Department of Environmental Engineering, Changwon National University, Kyungnam 641-773, Korea

Hak Sung LEE

School of Chemical Engineering, University of Ulsan, Ulsan 680-749, Korea

Nak Chang SUNG

Department of Environmental Engineering, Donga University, Pusan 604-714, Korea

(Manuscript submitted February 14, 2003; accepted March 13, 2003)

\begin{abstract}
Mixed-bed ion exchange(MBIE) performance to remove nitrate in surface and underground water was studied experimentally in continuous operation system under the various conditions. Data were collected using commercially available strong-base anion- exchange resin of $\mathrm{OH}^{\top}$ type and strong-acid cation-exchange resin of $\mathrm{H}^{+}$type. The breakthrough curves, obtained through the continuous column system and plotted as the ratio of effluent to influent concentration versus solution volume passed through the experimental column, gave some results about the effect of the mixed-bed ion exchange. The breakthrough curves verified that the mixed-bed ion exchange showed better performance than single-bed ion exchange by using cation-to-anion resin ratio of 2:1, 1:1, and 1:2. More cation resin showed better anion exchange performance than less cation resin with same amount of anion resin in the column. The effect of system parameter such as feed concentration was added. The results of this study could be scaled up and used as a design tool for the water-purification system of the drinking water treatment processes.
\end{abstract}

\section{Introduction}

Nitrate is so toxic especially to pregnant women and infants that the USEPA and Korean standards of $10 \mathrm{mg} \mathrm{NO}_{3}^{-}-\mathrm{N} / \mathrm{dm}^{3}$ or less in drinking water were established for the human health. Nitrate is a nutrient source for the growth-limiting factor to the causes of eutrophication. This causes a fatal damage to the sea food industries.

Nitrate removal from drinking water using ion exchange is economical and convenient and provides a suitable solution for small or medium sized water treatment plants containing comparably low nitrates level. Ion exchange is the most common process for public water supplies in the United States. Approximately 15 of these plants were operating in the United States during 1992.[1] Ion exchange systems are operated on demand, and 
utilizes the beneficial selective reversal which commonly occurs upon regeneration. [2]

Previously parametric studies were performed to present nitrate removal characteristics of anion exchange resins by Yoon et al.[3] The system parameters for the study were resin type, initial nitrate concentration, volumetric flow rate, column diameter, height, and temperature using batch and continuous column systems. The breakthrough curves obtained from the continuous column gave some results; with small column diameter or long column height, with low initial feed concentration, with low volumetric flow rate, or with high solution temperature, the nitrate removal for the typical macroporous type resin increase.

This paper extends the earlier research performed by Yoon et al., $[3,4]$ who studied the effect of system parameters on the performance of anion exchange for the nitrate removal to the mixed bed which uses ammonium as a counter ion.

\section{Experimental}

For the mixed-bed ion exchange process, $\mathrm{H}^{+}$-type cation exchange resin of Dowex Monosphere 650 C and $\mathrm{OH}$-type of Dowex Monosphere 550A provided by the Dow Chemical Company were well mixed in the glass column. These resins were grouped into Type II of strong acidic and basic resins. The physicochemical properties of the resins are shown in Table 1.

Table 1. Characteristic values of ion exchange resins

\begin{tabular}{|c|c|c|}
\hline \multirow{2}{*}{ Parameters } & Cation & Anion \\
\cline { 2 - 3 } & $\mathrm{H}^{+}$type & OH type \\
\hline Name & Monosphere 650C & Monosphere 550A \\
\hline Capacity & $1.90 \mathrm{meq} / \mathrm{cm}^{3}$ & $1.10 \mathrm{meq} / \mathrm{cm}^{3}$ \\
\hline Selectivity & $\mathrm{Na}^{+}-\mathrm{H}^{+} 1.13$ & $\mathrm{Cl}-\mathrm{OH} 22.0$ \\
\hline Water Retention Capacity & $46-51 \%$ & $44-50 \%$ \\
\hline Density & $50.0 \mathrm{lb} / \mathrm{ft}^{3}$ & $40.0 \mathrm{lb} / \mathrm{ft}^{3}$ \\
\hline Diameter & $0.65 \mathrm{~mm}$ & $0.59 \mathrm{~mm}$ \\
\hline Appearance & Hard, Black, Spherical Bead & Hard, White, Spherical Beads \\
\hline
\end{tabular}

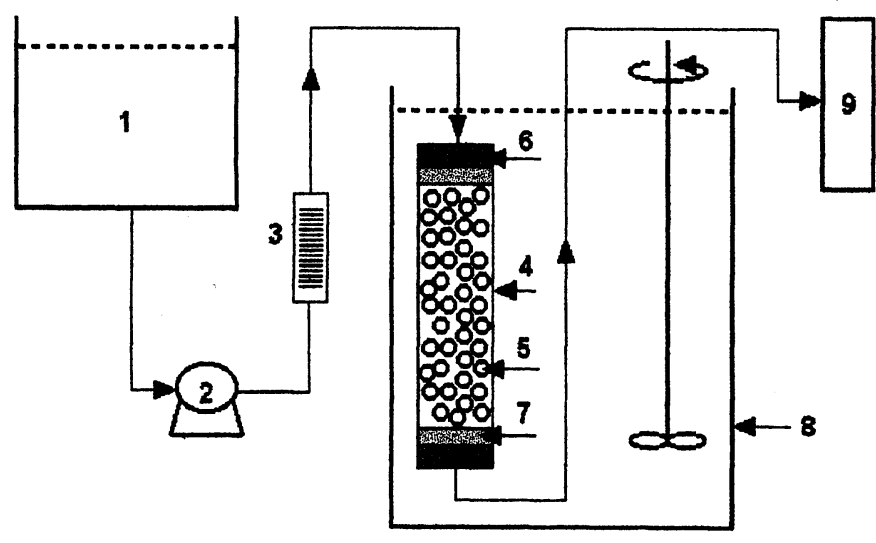
1. Feed storage
2. Pump 3. Flow meter
4. Glass column 5. Anion resin 6. Silicone stopper
7. Glass wool 8. Water bath 9. Ion chromatography

The experimental system in this study is mainly consisted of a packed-bed column, its accessory for feeding, water bath, feed storage, and ion chromatography for measuring effluent concentrations, and is shown in Fig. 1. The ion-exchange column was made with Pyrex glass to see the resin loaded inside the column. The continuous system has been performed to investigate the effects of the cation-to-anion resin ratio in the column, the difference and configurations between single- and mixed-bed on the breakthrough curves of nitrate.

Fig. 1. Schematic diagram of the continuous operation system. 


\section{Results and and Discussion}

The experiments were performed using continuous operation systems under the various conditions. The results of water sample analysis were described as the ratio of the effluent concentration $(C)$ to the feed concentration $\left(C_{0}\right)$ vs. solution volume passed through the experimental column. The performances of single-bed and mixed-bed ion exchange were compared as a function of cation-to-anion resin ratio and column configuration, and the effect of initial feed concentration was added.

Table 2. Experimental conditions of the column reactor

\begin{tabular}{|c|c|}
\hline Parameters & Conditions \\
\hline Feed Concentration & $\mathrm{NO}_{3}^{-} 9.92 \mathrm{mg} / \mathrm{dm}^{3}$ \\
& $\mathrm{NH}_{4}^{+} 2.88 \mathrm{mg} / \mathrm{dm}^{3}$ \\
\hline Feed Flow Rate & $10.8 \mathrm{dm}^{3} / \mathrm{h}$ \\
\hline Column Diameter & $12 \mathrm{~mm}$ \\
\hline Bed Depth & $20-70 \mathrm{~mm}$ \\
\hline Dry Resin Weight & $0.5-4.0 \mathrm{~g}$ \\
\hline Temperature & $15^{\circ} \mathrm{C}$ \\
\hline
\end{tabular}

In these experiments, a neutral salt solution of $\mathrm{NH}_{4} \mathrm{NO}_{3}$ was used as a feed solution. Used exchange resins were ionic forms of hydrogen and hydroxide for cation and anion exchange, respectively. Table 2 shows the experimental conditions of the system.

The results of the continuous column experiments are given in Figs. 2, 3, 4 and 5, from which we can see that the different levels of initial leakage of nitrate appear in the effluent of the exchange process. The initial leakages might be due to the insufficient contact time between the solution and the resin, which results from the high flow rate and the relatively short bed depth. These conditions were selected for the reasonable duration of the experimental runs. The performance of single-bed ion exchange and mixed-bed ion exchange for the removal of nitrate was compared in Figs. 2 and 3. It is observed in Fig. 2 that mixed-bed shows the steeper breakthrough curve at around 40 liter of treated volume in spite of the similar initial leakage.

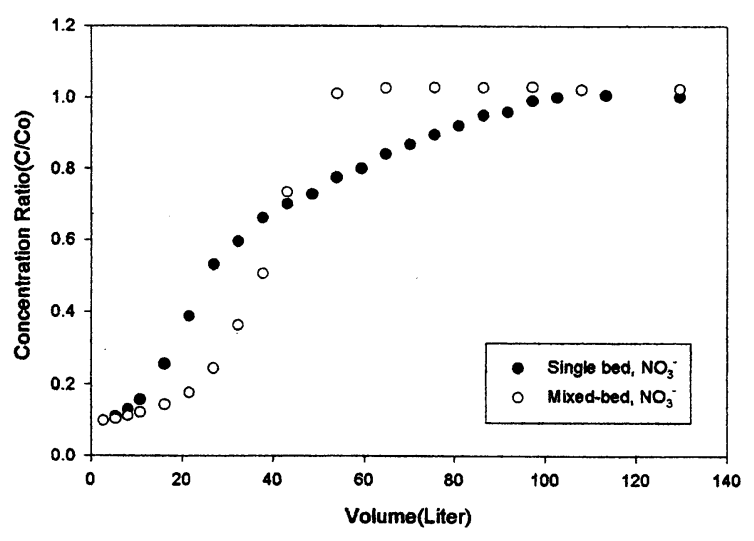

Fig. 2. Comparison of nitrate breakthrough curves with mixed- and single-bed

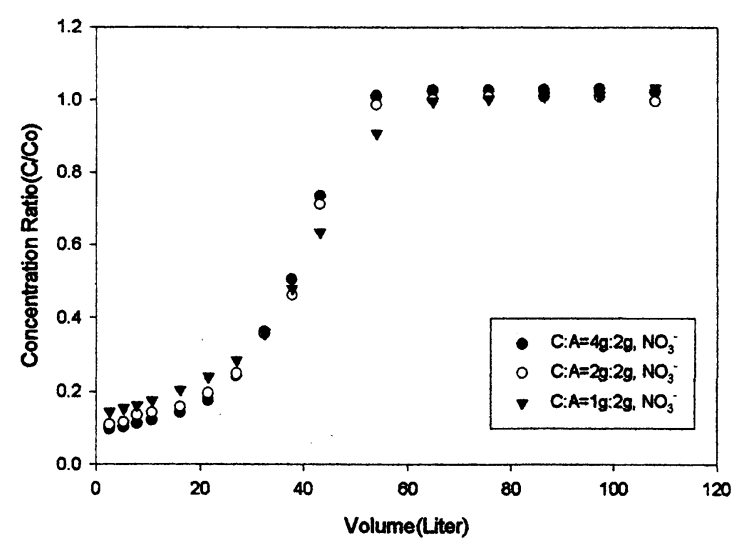

Fig. 3. Nitrate breakthrough curves of MBIE as a function of cation-to-anion resin ratio

The same amount of anion-exchange resin with smaller amount of cation-exchange resin produces a faster rate of hydroxide exchange than with greater amount of cation-exchange resin. This produces an aqueous phase with an alkaline $\mathrm{pH}$. However, Harries[5] showed that anion exchange is faster in an acidic medium than a neutral or alkaline medium since the mass-transfer coefficient of anion is higher at low $\mathrm{pH}$. Thus, anion exchange with higher cation-to-anion exchange resin ratio produces a steeper anion breakthrough curve. The experimental results in Figs. 2 and 3 show the same trends as Harries[5] claimed. The change of the 
cation-to-anion exchange resin ratio will change the $\mathrm{pH}$ of the aqueous phase within the bed. Thus, cation-to-anion resin ratio of $4 \mathrm{~g}$ to $2 \mathrm{~g}$ showed better nitrate breakthrough curve than that of $1 \mathrm{~g}$ to $2 \mathrm{~g}$ in Fig. 3 .

This can also be explained when we separate cation and anion beds in a column. Figure 4 shows the effect of column configuration. In this figure we have a better breakthrough curve when we pass the influent solution through cation bed first and anion bed later. This configuration makes the solution very acidic in the cation bed, and thus the higher mass-transfer coefficient of nitrate in the anion bed makes the sharper nitrate breakthrough curve. This configuration is even better than the mixed-bed for the removal of nitrate. The configuration with anion bed first and cation bed later shows a relatively broad nitrate breakthrough curve.

Figure 5 shows the effect of feed concentration on the nitrate breakthrough curve. As expected, the curves in Fig. 5 indicate the steeper slope and the faster breakthrough when the concentration increases. While the initial leakage also increases with the feed concentration from $0.8 \times 10^{-4} \mathrm{~N}$ to $1.6 \times 10^{-4} \mathrm{~N}$, it is almost constant regardless of the concentration from $1.6 \times 10^{-4} \mathrm{~N}$ to $3.2 \times 10^{-4} \mathrm{~N}$. The ratio of the treated solution volume, which is the left-hand side area of the breakthrough curves, agrees with the ratio of the feed concentration. Therefore, it can be said that the capacity of the resin is likely not to be changed by the variation of the initial feed concentration.

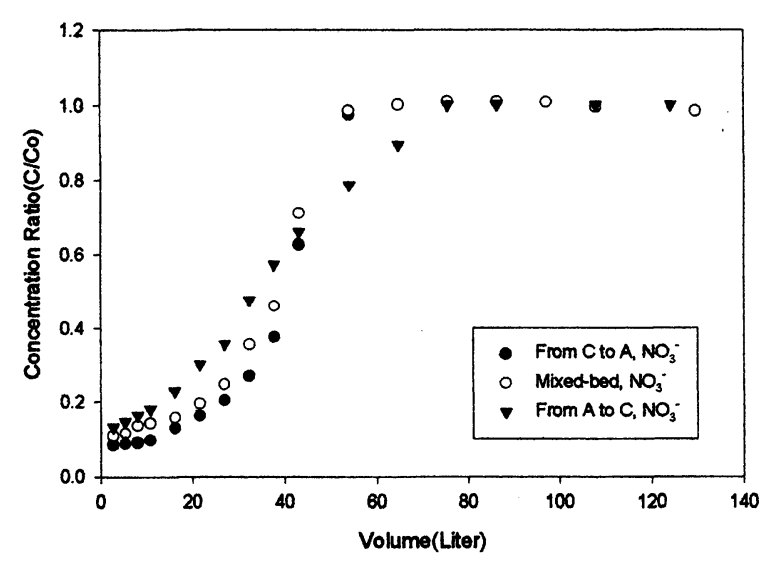

Fig. 4. Anion breakthrough curves with inlet flow cation to anion, anion to cation single bed and mixed bed

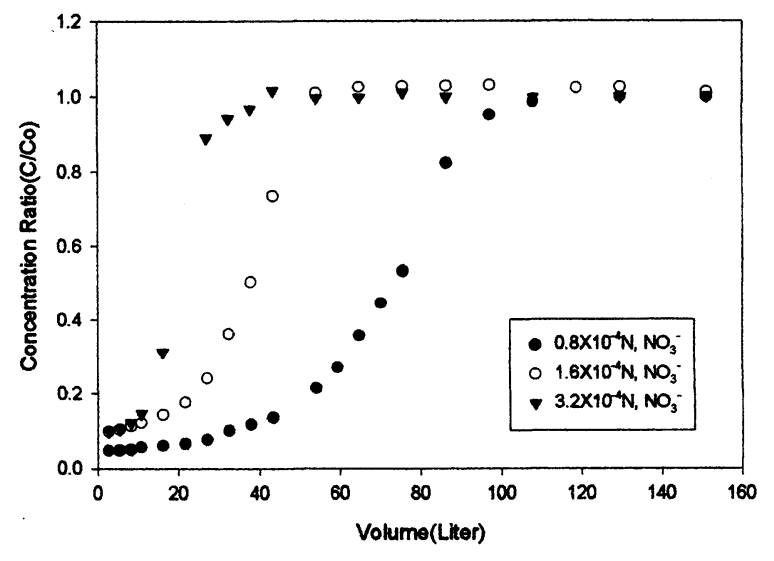

Fig. 5. Effect of initial concentration on nitrate breakthrough curves with MBIE

\section{Acknowledgement}

This research was supported by the Dongeui University Research Grants in 2001.

\section{References}

1) D. Clifford and X. Liu, Research and Technology, Journal AWWA, 135 (1993).

2) American Water Works Association, "Standard Methods for the Examination of Water and Wastewater," 18th Ed., Washington, D. C., 1992

3) T. Yoon, B. Noh, and B. Moon, Korean J. Chem. Eng., 17, 652-658 (2000)

4) T. Yoon, Z. H. Shon, G. Lee, B. Moon, B. Noh, and N. Sung, Korean J. Chem. Eng., 18, 170-177 (2001)

5) R. Harries, "The Role of pH in Ion Exchange Kinetics," in "Ion Exchange for Industry," (Ed. M. Street), Ellis Horwood Limited, England, 1988 